\title{
INFLUÊNCIA DA TEMPERATURA, SALINIDADE E LUZ SOBRE O CRESCIMENTO DO DINOFLAGELADO ALEXANDRIUM TAMARENSE (DINOFLAGELLATA, DINOPHYCEAE) DA PLATAFORMA CONTINENTAL ADJACENTE AO ESTUÁRIO DA LAGOA DOS PATOS
}

\author{
GRAZIELA DA R PERSICH ${ }^{1,2} \&$ VIRGINIA MARIA TAVANO GARCIA ${ }^{1,3}$ \\ ${ }^{1}$ Universidade Federal do Rio Grande - Instituto de Oceanografia - Laboratório de Ecologia do Fitoplâncton e Microorganismos Marinhos. \\ CxPostal: 474, 96201-900 - Rio Grande- RS, Brasil. ²gpersich@hotmail.com; ${ }^{3}$ docvmtg@furg.br
}

\begin{abstract}
O dinoflagelado Alexandrium tamarense (Lebour) Balech é uma das espécies potencialmente produtoras de saxitoxinas, as quais podem ser acumuladas ao longo da cadeia trófica e causar a síndrome denominada PSP (intoxicação paralisante por moluscos) em humanos, aves e mamíferos. A partir do isolamento de células móveis de $A$. tamarense proveniente da praia do Cassino, foi obtido um cultivo e a germinação de cistos de resistência coletados no sedimento originaram mais 12 cultivos. Todos estes foram mantidos sob condições controladas de temperatura $\left(20^{\circ} \mathrm{C}\right)$, intensidade luminosa $\left(350 \mu \mathrm{mol} \mathrm{m} \mathrm{m}^{-2} \mathrm{~s}^{-1}\right)$, salinidade $(31)$ e de nutrientes (meio de cultivo f/2, sem adição de silicato) até a realização dos experimentos. Foram realizados testes para verificação das condições ideais de crescimento, mediante aclimatação prévia às condições a serem testadas. Taxas de

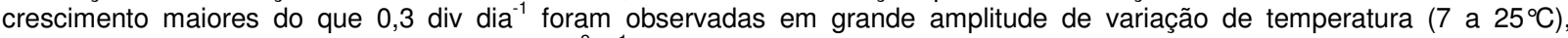
irradiância (valores maiores do que $80 \mu \mathrm{mol} \mathrm{m}^{-2} \mathrm{~s}^{-1}$ ) e salinidade (7 a 32). Porém, as taxas máximas de crescimento, de até 0,7 div dia ${ }^{-1}$, ocorreram sob condições mais restritas (temperatura entre 15 e $20^{\circ} \mathrm{C}$, irradiância acima de $200 \mu \mathrm{mol} \mathrm{m}^{-2} \mathrm{~s}^{-1}$ e salinidade entre 26 e 31). Estas taxas de crescimento estão entre os maiores valores encontrados para esta espécie, evidenciando a potencialidade que a população apresenta para desenvolver altas densidades celulares na costa sul do Brasil.
\end{abstract}

RESUMO

PALAVRAS CHAVE: Alexandrium tamarense, crescimento, temperatura, salinidade, luz, costa sul do Brasil

\begin{abstract}
Influence of temperature, salinity and light on growth rates of Alexandrium tamarense (Dinoflagellata, Dinophyceae) from the continental shelf close to Patos Lagoon estuary

The dinoflagellate Alexandrium tamarense (Lebour) Balech potentially produces saxitoxins which can be accumulated through the food web and cause a serious disease called PSP (Paralytic Shellfish Poisoning) in higher-level consumers, including humans, birds and mammals. There were obtained 13 strains through cysts germination and motile cells isolation that were kept in culture, under constant conditions of temperature $\left(20^{\circ} \mathrm{C}\right.$ ), light intensity $\left(\mathrm{ca} .350 \mu \mathrm{mol} \cdot \mathrm{m}^{-2} \cdot \mathrm{s}^{-1}\right)$, salinity $(31)$ and nutrients ( $\mathrm{f} / 2 \mathrm{medium}$ without silicate), until the experiments were carried out. Assays were performed to determine optimal environmental conditions for growth, after acclimation to respective test conditions. Although relatively high growth rates $\left(0.3\right.$ div.day $\left.^{-1}\right)$ were found under a wide range of temperature $\left(7\right.$ to $25^{\circ} \mathrm{C}$ ), light intensity (over $\left.80 \mu \mathrm{mol} . \mathrm{m}^{-2} . \mathrm{s}^{-1}\right)$ and salinity $\left(7\right.$ to 32 ), highest growth rates up to 0.7 div day ${ }^{-1}$ were measured under restricted ranges of temperature $\left(15\right.$ to $20^{\circ} \mathrm{C}$ ), light intensity (above $\left.150 \mu \mathrm{mol}^{-2} \mathrm{~m}^{-2} \mathrm{~s}^{-1}\right)$ and salinity $(26$ and 31 ). These growth rates are among the highest reported for Alexandrium tamarense under laboratory conditions and represent a potentiality for high cell concentrations during fast growth in the southern Brazilian coastal waters.
\end{abstract}

KEY WORDS: Alexandrium tamarense, growth, temperature, salinity, light. South Brazil

\section{INTRODUÇÃO}

As florações nocivas despertam interesse há séculos devido aos efeitos que podem produzir no ambiente. Estes efeitos incluem entupimento de brânquias de peixes, aspectos estéticos, formação de altos níveis de biomassa com conseqüente depleção de oxigênio e diminuição da penetração de luz na coluna de água. Outro tipo de efeito negativo destas florações pode ser a produção de toxinas, as quais podem matar diretamente peixes e moluscos ou causar intoxicações em humanos (Allen et al., 2006; Glibert et al. 2005).

Durante as últimas décadas verificou-se um aumento em nível mundial no número e tipo de florações de microalgas potencialmente tóxicas nas águas costeiras. Alguns fatores que devem ter atuado no aumento das florações nocivas são naturais, como a dispersão biológica natural das espécies.
Entretanto, outros fatores são de origem antrópica, como o aumento da carga nutritiva nas águas costeiras, dispersão por água de lastro, práticas agrícolas e de aqüicultura, sobrepesca e mudanças globais no clima (Glibert et al. 2005; Moore et al. 2008).

$\mathrm{Na}$ costa sul do Brasil, foram registrados quatro eventos de mortandade da fauna bentônica associados à presença de dinoflagelados. O primeiro ocorreu em abril de 1978, na praia do Hermenegildo, concomitante com a ocorrência de Gymnodinium sp. e associado à mortandade de moluscos e irritação respiratória em seres humanos (Machado, 1979). O segundo registro ocorreu em julho de 1981, também na praia do Hermenegildo, sendo detectada a presença de Gyrodinium cf. aureolum, e mortandade de poliquetas, moluscos, cnidários e equinodermas (Rosa e Buselato, 1981). Também foram constatados casos de irritação no aparelho respiratório humano, e 
eventuais casos de intoxicação de pessoas e animais domésticos que se alimentaram do molusco Mesodesma mactroides. O terceiro evento ocorreu em março de 1993, ao longo da praia do Cassino, com a presença dos dinoflagelados potencialmente nocivos Gyrodinium cf. aureolum (em maior concentração), Dinophysis acuminata e Noctiluca scintillans. Neste, foi verificada a mortandade da comunidade bentônica intermareal, atingindo principalmente os bivalves normalmente abundantes Mesodesma mactroides e Donax hanleyanus, bem como o crustáceo Emerita brasiliensis (Garcia et al., 1994; Odebrecht et al., 1995). O quarto evento ocorreu em dezembro de 1994, no Chuí e praia do Hermenegildo, quando se verificou mortandade massiva de Mesodesma mactroides, e de Donax hanleyanus em menor quantidade. $O$ mesmo foi observado na costa do Uruguai, próximo à fronteira com o Brasil, onde também foi detectada a presença de toxinas diarréicas (DSP) (Mendez, 1995). Neste local, foi verificada a presença dos dinoflagelados Dinophysis caudata, Dinophysis acuminata e várias espécies de Ceratium, principalmente Ceratium furca, além de silicoflagelados.

Dentre as espécies formadoras de florações tóxicas, destaca-se o dinoflagelado Alexandrium tamarense, cuja ocorrência, até poucas décadas, estava limitada ao Hemisfério Norte. O dinoflagelado A. tamarense é uma das espécies potencialmente produtoras de saxitoxinas, responsáveis pela síndrome conhecida como PSP (toxinas paralisantes por moluscos). Essas toxinas bloqueiam os canais de sódio, causando, em casos extremos, paralisia respiratória em humanos, mamíferos marinhos, aves e peixes.

A periodicidade com que as florações tóxicas de $A$. tamarense se repetem em algumas áreas e o aparecimento de toxicidade em outras previamente livres de florações, levaram à intensificação dos estudos da espécie em todo o mundo. Apesar disto, ainda restam dúvidas sobre a posição taxonômica do complexo Alexandrium tamarensis, o qual engloba as espécies: A. tamarense, $A$. catenella e $A$. fundyense. A distinção entre estas espécies está principalmente baseada na presença ou ausência do poro ventral na placa 1' apical, na morfologia geral da célula e na habilidade das células para formar cadeias com mais de duas células (Taylor, 1985; Balech, 1995). Interessantes discrepâncias vêm sendo observadas com a aplicação de diferentes métodos de identificação, como o seqüenciamento do DNA ribossomal, com o qual foi verificado que diferentes morfotipos do complexo A. tamarensis, provenientes de uma mesma área podem ser mais similares geneticamente do que populações morfologicamente idênticas, mas geograficamente isoladas (Scholin et al., 1994).

A análise genética de espécimens coletados na costa sul do Brasil, utilizando-se o método de fragmentos polimórficos obtidos com enzimas de restrição, bem como o seqüenciamento da região $D 1$ D2 do DNA ribossomal evidenciaram semelhanças entre as cepas sul brasileiras e as populações originárias da costa leste e oeste norte americana, além de semelhanças com outras cepas do complexo "tamarensis" da América do Sul (Persich et al., 2006). Contudo, estas cepas sul brasileiras formaram um subgrupo diferente dos demais conhecidos. Os resultados da análise genética evidenciam a provável origem comum entre estas populações. Os registros históricos vêm a corroborar com esta hipótese, pois eventos de florações tóxicas iniciaram no Chile em 1886 (Sengers, 1908), onde ocorre o morfotipo Alexandrium catenella, e na costa da Argentina e Uruguai em 1980 (Carreto et al., 1985 e Davison e Yentsch, 1985, respectivamente). Já na costa sul brasileira a espécie foi primeiramente detectada, em alta concentração $\left(2.10^{5} \mathrm{cel} \mathrm{L}^{-1}\right)$ apenas em 1996 (Odebrecht et al., 1997). Estes registros indicam que a dispersão da espécie na América do Sul está ocorrendo de sul para norte (Persich et al., 2006).

Altos valores de toxicidade (até $52.920 \mu \mathrm{g}$ STX eq. $100 \mathrm{~g}^{-1}$ de carne de mexilhão) foram registrados como conseqüência de florações de Alexandrium catenella no Chile (Guzmán et al., 2002). Na Argentina e Uruguai, as recorrentes florações de Alexandrium tamarense resultaram em concentração de saxitoxinas na carne de mexilhão, com valores de

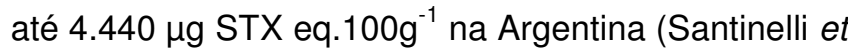
al., 2002) e $8.285 \mu \mathrm{g}$ STX eq. $100 \mathrm{~g}^{-1}$ no Uruguai (Méndez e Ferrari, 2002). Não há informação a respeito da concentração de saxitoxina na carne de moluscos quando ocorreram as altas concentrações de Alexandrium tamarense na costa sul brasileira. 
Entretanto, análises de saxitoxinas nas cepas provenientes desta área resultaram em concentrações de até 66.000 fgSTXeq cel $^{-1}$, o qual está entre os maiores valores de toxicidade obtidos em cultivos da espécie (Persich et al., 2006).

O potencial risco de intoxicação com saxitoxinas, associado às conseqüentes perdas econômicas com a suspensão do consumo e comercialização de recursos marinhos, motivaram diversos estudos a respeito das condições ambientais ideais ao crescimento de populações de $A$. tamarense (ex. Anderson, 1998; Anderson, 2004; Anderson e Wall, 1978; Anderson et al., 1983; Hamasaki et al., 1997; Su et al., 1993). Entretanto, observou-se variabilidade nas respostas fisiológicas, devido a variações genéticas, principalmente entre populações provenientes de diferentes áreas, sendo estas variações atribuídas ao isolamento geográfico e desenvolvimento independente das populações (Scholin et al., 1995). O fato de a espécie incluir uma fase sexuada no seu ciclo de vida propicia o intercâmbio gênico e garante uma maior variabilidade genética, em comparação com espécies que apresentam apenas reprodução assexuada. Portanto, é esperada certa variabilidade nas respostas às condições ambientais, mesmo dentro de uma mesma região, como constatados por Brand (1980). Consequentemente, as populações podem apresentar alta plasticidade e adaptabilidade a condições ambientais encontradas em diferentes áreas.

Os fatores envolvidos no desenvolvimento de florações de $A$. tamarense, como ocorre com os demais dinoflagelados, são complexos. A espécie produz cistos de resistência (hipnozigotos) como resultado do processo de reprodução sexuada, os quais afundam e são acumulados preferencialmente em fundos de lama, podendo permanecer viáveis por vários anos. Ao retornarem as condições ambientais favoráveis, pode haver germinação dos cistos e retomada do crescimento celular, originando novas florações da espécie no ambiente (Anderson, 1980). As principais funções dos cistos de resistência são, portanto, a manutenção da carga genética através da meiose, a proteção contra os fatores ambientais adversos, a propagação da população no ambiente, e a dispersão das espécies através de correntes (Dale, 1983).
O presente estudo pretende fornecer o conhecimento básico sobre as características fisiológicas de cepas locais da espécie, procurandose entender as variações nas taxas de crescimento ao longo de um gradiente de temperatura e sob diferentes condições de luz e salinidade. Com isto, espera-se oferecer subsídios ao desenvolvimento de futuros estudos a respeito de florações de Alexandrium tamarense na costa brasileira, para a qual os registros da espécie iniciaram somente em agosto de 1996, indicando uma recente dispersão da espécie.

\section{MATERIAL E MÉTODOS}

\section{Área de estudo}

A linha da costa no sul do Brasil é, caracteristicamente, sem reentrâncias, exposta à dinâmica de ondas, apresenta sedimento predominantemente arenoso e é classificada como "dissipativa a reflectiva", com relação à largura da zona de arrebentação, declividade e energia de ondas (Calliari et al., 1996). Apesar da predominância da fração areia, a antepraia (definida como a área desde a zona de quebra das ondas até o oceano aberto) apresenta grande heterogeneidade na distribuição dos sedimentos. Os depósitos de lama ocorrem próximo à desembocadura da Lagoa dos Patos e, devido à direção predominante de transporte da pluma de sedimentos da Lagoa ocorrer para o sul, os sedimentos finos são depositados ao sul desta área (Fachin, 1998). Como resultado da influência continental, a plataforma apresenta menor declividade, associada à presença de granulometria fina, resultando em uma combinação favorável ao desenvolvimento de uma abundante comunidade bentônica neste local (Calliari et al., 1996).

A plataforma continental ao sul do Brasil representa a área pesqueira mais importante da costa brasileira (Haimovici et al., 1989; Castello et al., 1990). A concentração de organismos bentônicos também é alta, sendo que na área intermareal os filtradores como Mesodesma mactroides e Emerita brasiliensis representam os maiores consumidores do fitoplâncton (Gianuca, 1998). A alta produção secundária encontrada nesta área é estimulada pela 
alta biomassa fitoplanctônica verificada na região. O fitoplâncton encontra-se principalmente representado pelas diatomáceas, porém ocorrem altas concentrações de dinoflagelados associadas à baixa energia de ondas e altos valores de intensidade luminosa na coluna de água (Odebrecht et al., 1997).

\section{Coleta e processamento das amostras de sedimento}

Amostras de camadas superficiais do sedimento foram coletadas em 43 pontos, em profundidades entre 5 e $28 \mathrm{~m}$, localizados entre as latitudes $32^{\circ} 04^{\prime}$ e $32^{\circ} 30^{\prime} \mathrm{S}$ e longitudes $51^{\circ} 49^{\prime} \mathrm{e}$ $52^{\circ} 10^{\prime} \mathrm{W}$ (Figura 1). As coletas foram realizadas entre março e novembro de 1997, e em março de 1999, utilizando-se amostrador de gravidade em condições de calmaria ou draga tipo Van-Veen em situações de mar agitado. Os dois centímetros superficiais do sedimento coletado em cada estação foram colocados em recipientes de vidro preenchidos até a borda com água do ambiente, vedados, mantidos ao abrigo da luz e armazenados em refrigerador a $4^{\circ} \mathrm{C}$. $\mathrm{O}$ processamento do material foi realizado conforme algumas modificações da metodologia descrita por Matsuoka e Fukuyo (1987). O primeiro centímetro superficial de sedimento de cada estação foi processado em sonificador Branson 250 durante 60 segundo a $100 \%$ e intensidade 4 . A lavagem do material, em duas peneiras com malhas de 20 e 80 $\mu \mathrm{m}$, possibilitou concentrar os cistos nesta faixa de tamanho.

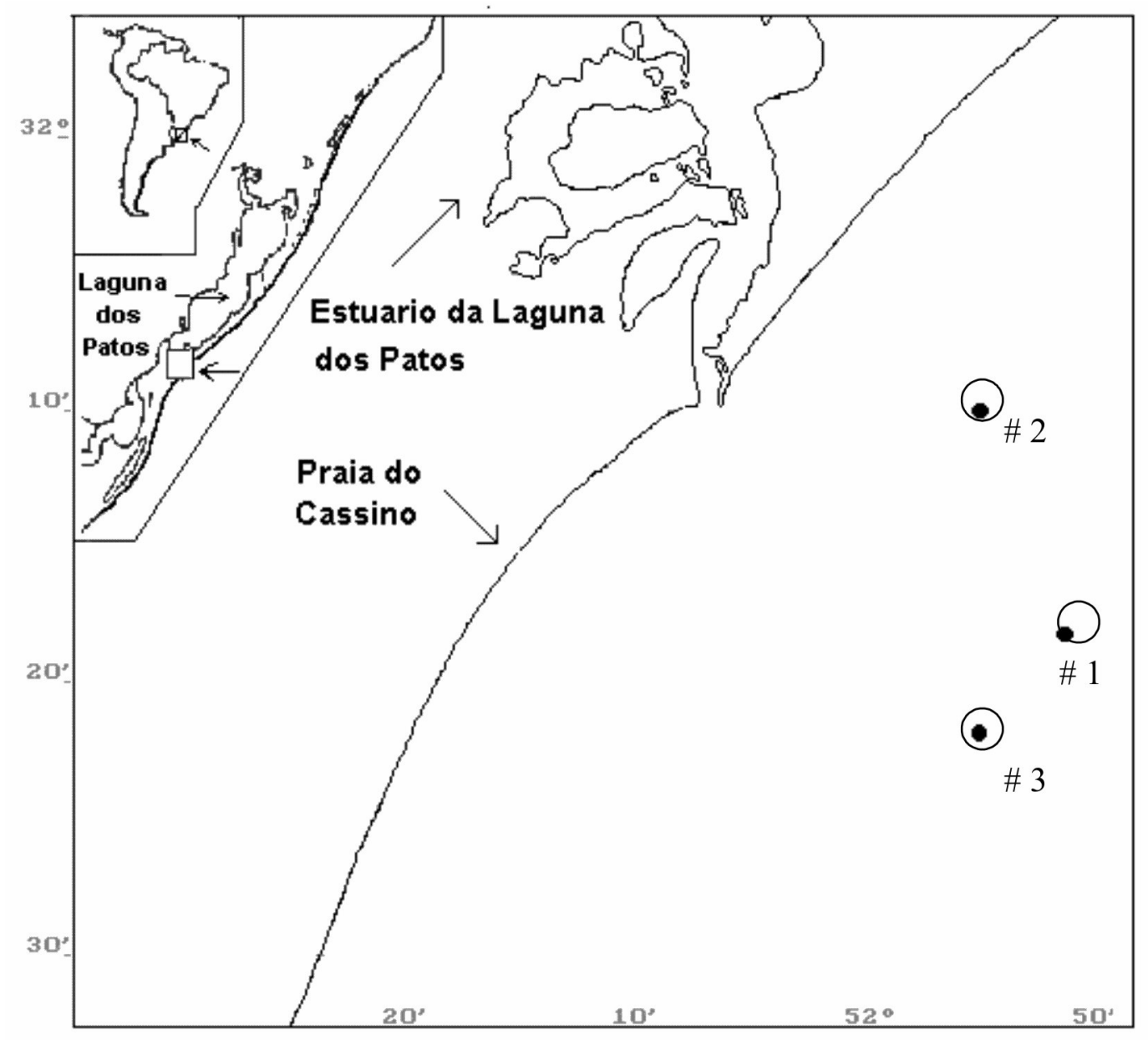

\section{Obtenção e manutenção dos cultivos}

O cultivo denominado de Cepa 6 foi obtido através da coleta de água de superfície em área rasa da Praia do Cassino, RS, em agosto de 1997, e posterior isolamento das células em microscópio comum, mediante suç̧ão com pipeta de pasteur e lavagem de cada célula em gotas do meio f/2.

Todas as demais cepas (12) foram obtidas mediante isolamento e germinação dos cistos 
provenientes do sedimento de fundo da praia do Cassino. Os cistos foram isolados em microscópio com capilar de vidro e colocados em cavidades individuais contendo $f / 2$, em placas utilizadas para cultivo de tecidos, seladas e mantidas a $20^{\circ} \mathrm{C}$ e irradiância de aproximadamente $250 \mu \mathrm{mol} \mathrm{m} \mathrm{m}^{-2} \mathrm{~s}^{-1}$. Após a germinação, as células móveis resultantes foram isoladas e desta forma foi adquirido um cultivo para cada cisto germinado, utilizando-se material proveniente de 3 estações de coleta. Os cistos provenientes da estação 2 , originaram as cepas $2 \mathrm{a}$, $2 \mathrm{~b}, 2 \mathrm{c}, 2 \mathrm{~d}, 2 \mathrm{e}, 2 \mathrm{f}$, e 2g; os cistos provenientes da estação 3 , originaram as cepas $3 a, 3 c$ e $3 d$. Para a estação 1, um único cisto germinou e deu origem aos dois clones denominados de "1a7" e "1a10". A localização das estações de coleta e respectivas concentrações de cistos de $A$. tamarense constam na Figura 1.

Os cultivos foram mantidos em meio $f / 2$ (Guillard e Ryther, 1962), com exceção da adição de silicato. A repicagem dos cultivos foi realizada em capela de fluxo laminar e sempre na fase exponencial de crescimento, monitorada pela leitura da fluorescência "in vivo" utilizando-se fluorímetro 10-AU Turner Designs.

\section{Identificação da espécie}

A identificação da espécie foi confirmada mediante concentração dos cultivos por centrifugação, fixação das células com formol a $5 \%$, lavagem da matéria orgânica com 0 detergente Triton $\mathrm{X}$ e adição de calcoflúor. Após este procedimento, as células foram observadas em aumento de 600 a 1000 vezes, para visualização do poro ventral, que é uma das características pelas quais se pode distinguir $A$. tamarense de $A$. fundyense. Apesar de presente, o poro ventral, na maioria dos casos, foi de difícil detecção, pois com o tempo de cultivo as tecas vão se tornando mais finas, dificultando a identificação de estruturas contidas nas mesmas (J. Larsen, com. pessoal).

\section{Cálculo das taxas de crescimento}

As taxas de crescimento foram obtidas a partir da leitura aproximadamente diária dos valores de fluorescência "in vivo", realizada em fluorímetro Turner Designs 10-AU. Inicialmente foi realizada análise de regressão linear e constatado alto grau de explicabilidade $\left(r^{2}\right.$ entre 0,79 e 0,94$)$ entre concentração celular em função da variação dos valores de fluorescência "in vivo". O aparelho foi previamente calibrado, tomando-se como branco o mesmo frasco de $25 \mathrm{ml} \mathrm{com} \mathrm{o} \mathrm{meio} \mathrm{f/2,} \mathrm{utilizados} \mathrm{em}$ todos os experimentos; os cultivos foram desenvolvidos sob as mesmas condições; as leituras foram sempre realizadas em triplicatas, no mesmo horário e mediante rígida padronização dos procedimentos de homogeneização e leitura.

Os valores de fluorescência $x$ tempo foram plotados em escala semilogarítmica, e exatamente os mesmos critérios foram adotados para encontrar o período de máximo crescimento ou crescimento exponencial, utilizado como base para os cálculos das taxas de crescimento e do número de divisões por dia, sendo a constante de crescimento $(\mathrm{Ke})$ calculada com a seguinte fórmula:

$$
\mathrm{Ke}=\frac{\ln \left(\mathrm{N}_{1} / \mathrm{N}_{0}\right)}{\mathrm{t}_{1}-\mathrm{t}_{0}}
$$

Sendo,

$N_{1}=$ valor de fluorescência no final da fase exponencial de crescimento

$\mathrm{N}_{0}=$ valor de fluorescência no início da fase exponencial de crescimento

$\mathrm{T}_{1}=$ idade do cultivo, em dias, até a fase final de crescimento exponencial

$\mathrm{T}_{0}=$ idade do cultivo, em dias quando iniciou a fase exponencial de crescimento

O número de divisões por dia (k) foi calculado convertendo-se o logarítmo neperiano a log na base 2, de acordo com a seguinte fórmula:

$$
\mathrm{k}=\frac{\mathrm{Ke}}{0,6931}
$$

Diferenças entre médias nas taxas de crescimento sob diversos tratamentos foram avaliadas através da análise de variância nas médias e após com o teste de Turkey para verificar as diferenças significativas. 


\section{Condições de temperatura, irradiância e salinidade}

Todos os experimentos relatados a seguir, testando-se as taxas de crescimento sob diferentes condições de crescimento dos cultivos, foram realizados no Instituto Oceanográfico de Woods Hole, no laboratório do Dr. Donald Anderson.

As condições ótimas para as variáveis ambientais estudadas foram obtidas a partir de experimentos realizados com a cepa $3 c$, mediante leituras aproximadamente diárias e em triplicata da fluorescência para cada tratamento. Esta cepa foi escolhida porque testes prévios evidenciaram valor de correlação entre densidade celular e fluorescência intermediário entre os obtidos para as outras cepas. O tempo de aclimatação dos cultivos às condições experimentais foi equivalente ao de três inoculações (aproximadamente 45 dias), e os experimentos foram realizados mantendo-se o mais constante possível as seguintes condições: irradiância, medida dentro dos frascos contendo os cultivos, utilizando-se irradiômetro digital com sensor esférico QSL -100 (aproximadamente $350 \mu \mathrm{mol} \mathrm{m} \mathrm{m}^{-2} \mathrm{~s}^{-1}$ ), salinidade (31), temperatura $\left(20^{\circ} \mathrm{C}\right)$ e fotoperíodo (14luz:10escuro). Estas condições foram adotadas como constantes em todos os experimentos, variando apenas nos experimentos onde eram as respectivas variáveis a serem analisadas.

\section{Temperatura}

A influência da temperatura no crescimento celular foi determinada mediante a utilização de uma barra de gradiente de temperatura (Watras et al., 1982), constituída por uma barra metálica, forrada com placas de isopor e contendo 20 colunas com quatro orifícios cada, onde eram inseridos os tubos com $25 \mathrm{ml}$ de cultivo. O gradiente de temperatura foi obtido através da conexão com um aquecedor em uma das extremidades, com um refrigerador na outra, e mediante a circulação de um líquido de refrigeração por toda a barra. As faixas de temperatura foram obtidas a partir de calibração dos dispositivos da barra, tentando-se obter os valores mais próximos possível das variações encontradas no ambiente. Os valores foram fixados somente após medições de temperatura, realizadas dentro de frascos com meio de cultivo, em diferentes horários durante várias semanas. As faixas utilizadas foram as seguintes: 26 a $27^{\circ} \mathrm{C}, 25$ a $26^{\circ} \mathrm{C}, 23$ a $25^{\circ} \mathrm{C}, 22$ a $24^{\circ} \mathrm{C}, 21$ a $23^{\circ} \mathrm{C}$, 20 a $22^{\circ} \mathrm{C}, 18$ a $20^{\circ} \mathrm{C}, 17$ a $19^{\circ} \mathrm{C}, 15$ a $17^{\circ} \mathrm{C}, 14$ a $16^{\circ} \mathrm{C}, 13$ a $15^{\circ} \mathrm{C}, 11$ a $14^{\circ} \mathrm{C}, 9$ a $12^{\circ} \mathrm{C}, 7$ a $10^{\circ} \mathrm{C}, 6$ a $9^{\circ} \mathrm{C}$ e 4 a $6^{\circ} \mathrm{C}$. A irradiância para este experimento foi obtida mediante a colocação de lâmpadas fluorescentes e combinação de malhas no fundo transparente da caixa. Primeiramente, a barra foi ajustada para valores de irradiância entre 80 e 100 umol $\mathrm{m}^{-2} \mathrm{~s}^{-1}$, considerada representativa de uma situação média ao longo do dia, no inverno, na coluna de água da Praia do Cassino. Entretanto, o crescimento foi praticamente nulo, e então se optou por maiores valores, entre 261 e $322 \mu \mathrm{mol} \mathrm{m} \mathrm{m}^{-2} \mathrm{~s}^{-1}$. Estes valores foram obtidos após inúmeras tentativas de homogeneização das intensidades luminosas em todos os orifícios contendo os tubos-testes, sendo que os tubos com intensidades superiores ou inferiores a estes valores não foram utilizados. Para minimizar possíveis variações de irradiância entre os diferentes orifícios utilizados, os frascos eram diariamente mudados de posição dentro de cada coluna representativa dos diferentes tratamentos.

\section{Irradiância}

Os valores de irradiância testados (380, 180, 150, 100, 80 e $50 \mu \mathrm{mol} \mathrm{m} \mathrm{m}^{-2} \mathrm{~s}^{-1}$ ) foram obtidos mediante combinações das malhas cinza e preta. Estas envolviam os suportes com os cultivos, os quais tiveram as suas posições na câmara incubadora trocadas diariamente. A câmara incubadora era formada por várias prateleiras, abaixo das quais era posicionada a fonte luminosa, constituída por tubos fluorescentes de luz branca.

\section{Salinidade}

As diferentes salinidades utilizadas $(31,25,18$, 14,12 e 5) foram obtidas mediante diferentes proporções de diluição da água do mar com água destilada e deionizada. O procedimento de adição de nutrientes e preparo do meio foi idêntico aos outros tratamentos. 


\section{RESULTADOS}

\section{Crescimento sob diversas condições}

Os experimentos de taxas de crescimento nas condições padrão foram realizados para a cepa 6 , obtidas a partir do isolamento de células móveis da coluna de água, e para as cepas 1a10, 2a e 3a, provenientes da germinação dos cistos das estações 1, 2 e 3, respectivamente. Valores médios e desvios padrões, calculados com base nas taxas de crescimento, constam na Tabela 1.

Tabela 1 - Valores médios e desvio padrão das taxas de crescimento (k) obtidas em triplicatas, a partir de dados da Figura 2. F0 e $F=$ valores médios de fluorescência inicial e final, respectivamente. T0 e $T=$ tempo inicial e final, em dias, respectivamente. Os asteriscos indicam as médias estatisticamente semelhantes $(p<0,05)$, conforme o teste de Tukey.

\begin{tabular}{|lllllrl|}
\hline \multicolumn{2}{|c}{ Cepa } & K & F & F0 & T \\
\hline 1a10 & a & $\mathbf{0 , 7 2}(\mathbf{0 , 0 1 3 )}$ & 18,20 & 0,93 & 9 & 3 \\
2a & b & $\mathbf{0 , 5 9}(\mathbf{0 , 0 2 2 )}$ & 12,55 & 1,08 & 8 & 2 \\
3a & c & $\mathbf{0 , 6 0 * ( 0 , 0 0 6 )}$ & 12,50 & 1,03 & 8 & 2 \\
C6 & d & $\mathbf{0 , 2 5}(\mathbf{0 , 0 1 4 )}$ & 17,25 & 1,02 & 19 & 3 \\
\hline
\end{tabular}

A taxa de crescimento $(k)$ variou entre $0,25 \mathrm{e}$ 0,72 div dia ${ }^{-1}$, para as cepas $\mathrm{C} 6$ e 1 , respectivamente, com valores semelhantes e intermediários, de aproximadamente $0,60 \mathrm{div}^{\mathrm{dia}}{ }^{-1}$ para as cepas 2 e 3 .
As taxas de crescimento, calculadas para os períodos de crescimento exponencial da cepa 3c, nas 16 faixas de temperatura testadas, constam na Tabela 2.

\section{Temperatura}

Tabela 2 - Valores médios (k) e desvio padrão (s) das taxas de crescimento, obtidos em triplicatas, para a cepa 3c, cultivada sob diferentes faixas de temperatura. As letras sobre as taxas de crescimento referem-se aos valores estatisticamente semelhantes $(p<0,05)$, de acordo com o teste de Tukey.

\begin{tabular}{|c|c|c|c|c|c|c|c|}
\hline Temperatura & & K & $\mathrm{s}$ & $F$ & F0 & $\mathrm{T}$ & T0 \\
\hline $26-27$ & $a$ & 0,00 & 0,000 & 0,00 & 0,00 & & \\
\hline $25-26 \mathrm{k}$ & $b$ & $0,24^{\mathrm{op}}$ & 0,040 & 1,48 & 0,75 & 8 & 4 \\
\hline $23-25 \mathrm{c}$ & c & $\mathbf{0}, \mathbf{3} 5^{\text {dejkmn }}$ & 0,009 & 10,18 & 0,56 & 13 & 1 \\
\hline $22-24 \mathrm{c}$ & $d$ & $0,39^{\text {cejklm }}$ & 0,005 & 14,08 & 0,55 & 13 & 1 \\
\hline $21-23 \in$ & e & $0,39^{\text {cejklm }}$ & 0,007 & 8,31 & 0,55 & 11 & 1 \\
\hline $20-22 f$ & $f$ & $0,40^{\text {hil }}$ & 0,021 & 12,57 & 0,75 & 10 & 1 \\
\hline $18-20 \mathrm{~g}$ & $g$ & $0,51^{h}$ & 0,020 & 10,73 & 0,62 & 9 & 1 \\
\hline $17-19 \mathrm{r}$ & $\mathrm{h}$ & $0,47^{\mathrm{fgil}}$ & 0,027 & 11,28 & 0,60 & 10 & 1 \\
\hline $15-17 \quad \mathrm{i}$ & $\mathrm{i}$ & $0,46^{\text {fhl }}$ & 0,011 & 11,30 & 0,65 & 10 & 1 \\
\hline 14-16 j & j & $0,35^{\text {cdlkmn }}$ & 0,024 & 13,77 & 0,59 & 14 & 1 \\
\hline $13-15 k$ & $\mathrm{k}$ & $0,39^{\text {cdejlmn }}$ & 0,010 & 12,17 & 0,64 & 12 & 1 \\
\hline $11-14$ । & I & $0,44^{\text {defhik }}$ & 0,019 & 10,77 & 0,69 & 10 & 1 \\
\hline $9-12 n$ & $\mathrm{~m}$ & $0,36^{\text {cdejkn }}$ & 0,007 & 12,50 & 0,64 & 13 & 1 \\
\hline $7-10 r$ & $\mathrm{n}$ & $0,33^{\mathrm{cjkm}}$ & 0,016 & 10,51 & 0,56 & 14 & 1 \\
\hline $6-9 c$ & 0 & $0,23^{b p}$ & 0,002 & 14,57 & 0,73 & 20 & 1 \\
\hline $4-6 p$ & $p$ & $0,20^{\text {bo }}$ & 0,010 & 10,88 & 0,57 & 22 & 1 \\
\hline
\end{tabular}


Os resultados mostram que a redução nas taxas de crescimento foi semelhante para os valores extremos de temperaturas testados neste experimento $\left(4\right.$ a $\left.9^{\circ}\right)$ e $25^{\circ} \mathrm{C}$. Acima de $25^{\circ} \mathrm{C}$ a taxa de crescimento foi nula. Apesar da grande amplitude de tolerância da cepa às variações de temperatura, evidenciada pelas taxas de crescimento relativamente elevadas (acima de 0,33 div $\operatorname{dia}^{-1}$ ), que ocorreram entre as temperaturas de $7^{\circ} \mathrm{C}$ e $25^{\circ} \mathrm{C}$, valores significativamente maiores $\left(0,46\right.$ a 0,51 div dia $\left.^{-1}\right)$ ocorreram em faixa mais restrita, de $15^{\circ} \mathrm{C}$ a $20^{\circ} \mathrm{C}$, respectivamente.

\section{Irradiância}

As taxas de crescimento (k), obtidas para cada irradiância testada, constam na Tabela 3. A menor taxa de crescimento $\left(0,17\right.$ div dia $\left.^{-1}\right)$ ocorreu no menor valor de irradiância testado $\left(50 \mu \mathrm{mol} \mathrm{m} \mathrm{m}^{-2} \mathrm{~s}^{-1}\right)$. A partir de $150 \mu \mathrm{mol} \mathrm{m} \mathrm{m}^{-2} \mathrm{~s}^{-1}$, foram atingidos altos valores de taxas de crescimento (acima de 0,54 div dia $^{-1}$ ).

Tabela 3 - Valores médios (k) e desvio padrão (s) das taxas de crescimento, obtidos em triplicatas para a cepa 3c. As letras sobre as médias referem-se aos valores estatisticamente semelhantes $(p<0,05)$, de acordo com o teste de Tukey.

\begin{tabular}{|rlrrrrr|}
\hline Luz & $\mathbf{k}$ & $\mathrm{S}$ & $\mathrm{F}$ & $\mathrm{F} 0$ & $\mathrm{~T}$ & T0 \\
\hline $50 \mathrm{a}$ & $\mathbf{0 , 1 7}$ & 0,00 & 6,6 & 1,0 & 25 & 9 \\
$80 \mathrm{~b}$ & $\mathbf{0 , 4 0 ^ { \mathrm { c } }}$ & 0,01 & 12,2 & 0,7 & 11 & 1 \\
$100 \mathrm{c}$ & $\mathbf{0 , 3 5}^{\mathbf{b}}$ & 0,01 & 15,6 & 0,7 & 14 & 1 \\
$150 \mathrm{~d}$ & $\mathbf{0 , 5 4}^{\mathrm{e}}$ & 0,00 & 12,2 & 0,6 & 9 & 1 \\
$180 \mathrm{e}$ & $\mathbf{0 , 5 4 ^ { \mathrm { d } }}$ & 0,01 & 11,2 & 0,5 & 9 & 1 \\
$380 \mathrm{f}$ & $\mathbf{0 , 6 3}$ & 0,01 & 14,5 & 0,7 & 8 & 1 \\
\hline
\end{tabular}

\section{Salinidade}

As taxas de crescimento, calculadas para a cepa 3c, cultivada sob diferentes condições de salinidade são mostradas na Tabela 4. Foi observado crescimento elevado em uma ampla faixa de valores de salinidade (14 a 32). Entretanto, as maiores taxas de crescimento (aproximadamente 0,7 div $\mathrm{dia}^{-1}$ ) ocorreram nas maiores salinidades testadas (26 e 32). Valores relativamente elevados e estatisticamente similares (aproximadamente 0,56 div $\mathrm{dia}^{-1}$ ) ocorreram nas salinidades de 14 e 18. A menor taxa de crescimento $(0,31)$ foi verificada na salinidade de 7 , sendo que em 5 de salinidade não houve crescimento.

Tabela 4 - Valores médios (k) e desvio padrão (s), obtidos em triplicatas para a cepa 3c. As letras sobre as médias representam os valores estatisticamente semelhantes $(p<0,05)$, calculados a partir do teste de Tukey.

\begin{tabular}{|cccccccc|}
\hline Salinidade & & $\mathbf{k}$ & $\mathrm{S}$ & $\mathrm{F}$ & $\mathrm{F} 0$ & $\mathrm{~T}$ & $\mathrm{T0}$ \\
\hline $32 \mathrm{a}$ & $\mathbf{0 , 7 0 ^ { \mathbf { b } }}$ & 0,04 & 10,9 & 0,598 & 7 & 1 \\
$26 \mathrm{~b}$ & $\mathbf{0 , 7 1 ^ { \mathbf { a } }}$ & 0,01 & 11,4 & 0,602 & 7 & 1 \\
$18 \mathrm{c}$ & $\mathbf{0 , 5 7 ^ { \mathbf { d } }}$ & 0,01 & 11,4 & 0,487 & 9 & 1 \\
$14 \mathrm{~d}$ & $\mathbf{0 , 5 5 ^ { \mathbf { c } }}$ & 0,02 & 10,5 & 0,509 & 9 & 1 \\
$7 \mathrm{e}$ & $\mathbf{0 , 3 1}$ & 0,02 & 1,4 & 0,600 & 7 & 3 \\
$5 \mathrm{f}$ & $\mathbf{0 , 0 0}$ & 0,00 & - & & - & - \\
\hline
\end{tabular}

\section{DISCUSSÃO}

\section{Condições favoráveis ao crescimento de Alexandrium tamarense}

A variação nas taxas de crescimento, verificada para as diferentes cepas de $A$. tamarense, obtidas da mesma área e cultivadas sob as mesmas condições, podem refletir o grau de variabilidade fisiológica das mesmas. No entanto, a menor taxa de crescimento verificada para a cepa $6\left(0,25 \mathrm{div}^{\left.\mathrm{dia}^{-1}\right)}\right.$ pode ser conseqüência do seu maior tempo de cultivo, pois esta cepa foi mantida em laboratório por mais tempo. 
Já as outras cepas foram obtidas poucos meses antes da realização dos experimentos, através da germinação dos cistos de resistência. Outro fator que deve ter contribuído para esta diferença com relação às demais cepas foi o método utilizado para a sua obtenção. As células não foram isoladas imediatamente, mas sim mantidas em incubadora durante aproximadamente um mês. A mesma tendência foi verificada por Brand et al. (1981), que observou as menores taxas de crescimento em cepas isoladas somente após um mês de manutenção em incubadora, com relação a outras cepas provenientes do mesmo ambiente, mas que foram isoladas imediatamente após a coleta. A principal hipótese do autor para explicar este fato é que podem ter ocorrido mudanças devido à pressão de seleção em laboratório.

$\mathrm{Na}$ Tabela 5 são comparadas as condições ideais para o crescimento de $A$. tamarense verificadas neste estudo com cepas ou populações naturais provenientes de outras localidades.

Tabela 5 - Taxa de crescimento $\left(\mathrm{k}\right.$, divisões dia $\left.^{-1}\right)$ de $A$. tamarense proveniente de diferentes áreas geográficas e condições ambientais.

\begin{tabular}{|c|c|c|c|c|c|c|}
\hline ÁREA & $\begin{array}{l}\text { Temp } \\
\left({ }^{\circ} \mathrm{C}\right) .\end{array}$ & $\begin{array}{l}\text { Luz } \\
\left(\mu \mathrm{mol} \cdot \mathrm{m}^{-2} \cdot \mathrm{s}^{-1}\right)\end{array}$ & Salinidade & $\mathrm{k}\left(\right.$ div. dia $^{-1}$ ) & Observação & Ref. \\
\hline Sul do Brasil & $15-20$ & $>200$ & $26-31$ & $0,46-0,70$ & Cultivo & Pres.Trab. \\
\hline Argentina & 1820 & & & $0,3-0,6$ & Cultivo & 2 \\
\hline Argentina & 812 & & $>30$ & & Pop. natural & 3,4 \\
\hline Uruguai & 1115 & & $16-33,5$ & & Pop. natural & 5,6 \\
\hline EUA- NE & & & 30,5 & 0,36 & Cultivo & 7 \\
\hline EUA- NE & $13-23$ & & & 0,44 & Cultivo & 8 \\
\hline EUA- NE & $11-22$ & $150-200$ & & 0,6 & Cultivo & 9 \\
\hline Japão & $5-10$ & & $31,5-33$ & & Pop. natural & 10 \\
\hline Coréia & 17 & & $25-30$ & 0,6 & Cultivo & 11 \\
\hline Coréia & 15 & & & & Pop. natural & 11 \\
\hline Canadá & $15-19$ & & $19-20$ & 0,41 & Cultivo & 12 \\
\hline Tailândia & & & $10-35$ & & Cultivo & 13 \\
\hline
\end{tabular}

Referências: 1 - Presente trabalho; 2 - Benavides et al., 1983; 3,4 - Carreto et al., 1985; Carreto et al., 1998; 5,6 - Mendéz et al., 1996; Brazeiro et al., 1997; 7 - White, 1978; 8 - Watras et al, 1982; 9 - Anderson et al. 1984; 10 - Shimada et al., $1996 ; 11$ - Kim et al., $1996 ; 12$ Prakash, 1967; 13 - Su e Liau, 1993.

Apesar dos máximos valores de crescimento verificados no presente trabalho terem ocorrido nestas estreitas faixas de variação, valores relativamente elevados ocorreram em uma ampla faixa de variação das condições ambientais analisadas. Por exemplo, as taxas de crescimento

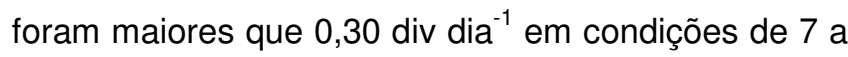
$25^{\circ} \mathrm{C}$ de temperatura, intensidades luminosas maiores do que $80 \mu \mathrm{mol} \mathrm{m} \mathrm{m}^{-2} \mathrm{~s}^{-1}$ e salinidade maior do que 7. A faixa ótima de temperatura observada no presente trabalho $\left(15^{\circ} \mathrm{C}\right.$ a $\left.20^{\circ} \mathrm{C}\right)$ é verificada na área costeira do sul do Brasil, principalmente durante o final de inverno/ início da primavera e outono. A análise das principais variáveis envolvidas na formação de florações da espécie na costa da Argentina e Uruguai mostra temperatura ambiental inferior à verificada como ideal no presente trabalho. $\mathrm{Na}$ costa da
Argentina, as máximas concentrações celulares foram registradas entre aproximadamente $8^{\circ} \mathrm{C}$ e $12^{\circ} \mathrm{C}$ e salinidade superior a 30 (Carreto et al., 1985; Andrinolo et al., 1999). Já em estudos de laboratório, foram medidas taxas de crescimento entre 0,3 e 0,6 div dia $^{-1}$ em cepas provenientes dessa área $e$ mantidas entre 18 e $20^{\circ} \mathrm{C}$ (Benavides et al., 1983). $\mathrm{Na}$ costa do Uruguai, as maiores concentrações celulares registradas ocorreram entre $11^{\circ} \mathrm{C}$ e $15^{\circ} \mathrm{C}$ e salinidade de 16 a 33,5 (Mendéz et al., 1996; Brazeiro et al., 1997).

Em revisão realizada por Anderson (1998), as máximas taxas de crescimento verificadas para a espécie estão entre 0,5 e 0,7 div dia $^{-1}$. Estudos realizados na costa nordeste da América do Norte, no Canadá e Estados Unidos mostraram máximas taxas de crescimento celular pouco superiores a 0,6 div dia ${ }^{-1}$ 
(Anderson et al., 1984), com valores entre 0,20 e 0,55 div $\mathrm{dia}^{-1}$ verificados durante florações na costa do Canadá (Fauchot et al. 2005). Estes máximos são coincidentes com o máximo valor encontrado para uma cepa proveniente da Coréia (Kim et al., 1996). Portanto, os máximos valores verificados no presente trabalho, de até 0,7 div lia $^{-1}$, estão entre os maiores valores observados para a espécie, demonstrando a potencialidade das populações provenientes do sul do Brasil para atingir grandes concentrações celulares no ambiente.

Com base na Tabela 5, percebe-se as que as condições de salinidade onde ocorre crescimento de A. tamarense apresentam grande amplitude de variação em diferentes populações. As cepas provenientes do sul do Brasil apresentaram maior taxa de crescimento nos máximos de salinidade testados. O mesmo padrão foi verificado em $A$. tamarense (referido como Gonyaulax excavata) proveniente da área costeira de Massachusetts, nordeste dos EUA, cujo máximo crescimento ocorreu entre 20 e 40 de salinidade (White, 1978). Da mesma forma, o crescimento máximo de uma cepa proveniente do sul da Coreia ocorreu a 25, seguido por 30, sendo que abaixo de 15 de salinidade não houve crescimento (Kim et al., 1996). Watras et al. (1982), comparando o crescimento em diferentes faixas de temperatura, encontraram maiores valores para 25 do que para 32 de salinidade. Valores intermediários de salinidade foram verificados para cepas provenientes da Baia de Fundy, Canadá (Prakash, 1967). A maior amplitude de tolerância à salinidade (5 a 40) e a mais ampla faixa ótima para o crescimento (10 a 35) foram verificadas para uma cepa proveniente da Tailândia (Su et al. 1993).

A faixa ótima de temperatura para 0 crescimento celular, verificada neste trabalho $\left(15^{\circ} \mathrm{C}\right.$ a $20^{\circ} \mathrm{C}$ ) é próxima aos valores encontrados para a Baía de Fundy (Prakash, 1967), Golfo de Maine (Yentsch et al., 1975) e Coreia (Kim et al., 1996). Taxas máximas de crescimento celular ocorreram em maiores amplitudes de variação de temperatura em cepas provenientes da costa nordeste dos EUA (Watras et al., 1982). Entretanto, cepas provenientes dessa região não apresentaram crescimento abaixo de $7^{\circ} \mathrm{C}$ (Anderson et al., 1984), temperatura pouco superior à menor faixa de temperatura testada no presente trabalho $\left(4-6^{\circ} \mathrm{C}\right)$ e que resultou em baixo crescimento. Ocorrência de florações sob condições de baixas temperaturas (5 a $10^{\circ} \mathrm{C}$ ) foram detectadas no sudoeste do Japão (Shimada et al., 1996). Com relação à intensidade luminosa, os resultados obtidos para cepas do nordeste dos EUA foram semelhantes aos verificados neste trabalho (máxima taxa de crescimento de 0,6 div dia ${ }^{-1}$ e saturação a $150-200$ $\mu \mathrm{mol} \mathrm{m}^{-2} \mathrm{~s}^{-1}$ ) (Anderson et al. 1994).

A análise dos resultados obtidos no presente trabalho e a comparação com outros estudos da espécie evidenciam o caráter euritérmico e eurihalino da espécie, que justificam a sua ampla distribuição mundial (Taylor, 1985). Os resultados mais contrastantes ocorrem com relação à salinidade, e refletem o maior grau de adaptabilidade das populações aos regimes locais de salinidade. Florações de $A$. tamarense no ambiente costeiro são estimuladas em condições ambientais ideais, embora esta espécie normalmente não apresente taxas de crescimento muito elevadas, comparadas com diatomáceas, por exemplo, e, portanto, também não desenvolva altas concentrações celulares, dificilmente formando florações monoespecíficas (Anderson, 1998). Consequentemente, as florações da espécie estão associadas à manutenção de taxas de crescimento intermediárias, bem como a um declínio do crescimento de outras espécies. A ocorrência de processos físicos de concentração celular, tais como frentes oceânicas é muito importante para a acumulação de grandes concentrações de células (Yentsch et al., 1975).

\section{Ocorrência Potencial de Florações Tóxicas de A. tamarense}

O valor máximo de toxicidade verificado nas cepas provenientes do sul do Brasil, de

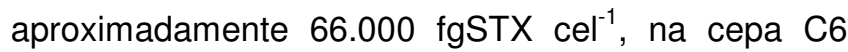
(Persich et al., 2006), pode ser considerado como relativamente elevado, pois está entre os maiores valores reportados para cepas mantidas em cultivos. As saxitoxinas, ingeridas diretamente através da pastagem das células tóxicas presentes no fitoplâncton, são acumuladas nos animais filtradores, até a posterior transferência através da cadeia trófica. Em cada etapa, a toxicidade resultante depende da sensibilidade do organismo às toxinas, e do grau de 
retenção e modificação que ocorrem nas saxitoxinas no interior do organismo. A sensibilidade dos consumidores à presença de saxitoxinas é variável, e os mexilhões normalmente são pouco sensíveis. Estes acumulam concentrações de toxinas que podem ultrapassar o limite de concentração considerado como de segurança para o consumo humano (Hall et al., 1990). O nível adotado varia com a legislação local, porém vários países, dentre eles Estados Unidos, consideram o valor de $80 \mu \mathrm{gSTX}$ $100 \mathrm{~g}^{-1}$ de carne de molusco como a concentração máxima permitida para a comercialização e consumo. As razões para a adoção deste critério baseiam-se fundamentalmente na verificação de que os sintomas clínicos da presença da toxina iniciam após o consumo de 200 a $600 \mu \mathrm{g}$ de toxina e considerandose um consumo diário $1 \mathrm{~kg}$ de moluscos, que representa aproximadamente $250 \mathrm{~g}$ de carne. Vários outros países, como a Itália, preocupados com os potentes efeitos das toxinas nas bombas de cálcio, sódio e potássio em seres humanos, adotaram o nível

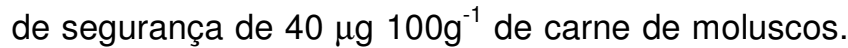
Apesar da presença de sintomas nestas concentrações relativamente baixas, a dose letal de saxitoxinas para humanos está entre $1 \mathrm{e} 2 \mathrm{~g}$ da toxina (Premazzi e Volterra, 1993).

A máxima concentração celular de Alexandrium tamarense verificada na Praia do Cassino, de $2.10^{5}$ cel $I^{-1}$ (Odebrecht et al. 1997), é característica de florações da espécie no ambiente (aproximadamente $2,5.10^{5} \mathrm{cel} \mathrm{I}^{-1}$, de acordo com Chebib et al., 1993). Existem, entretanto, registros de concentrações extremamente elevadas, como a verificada por Carreto et al. (1986), na Península Valdés, Argentina, em área de frente oceanográfica $\left(1,8.10^{6} \mathrm{cel} \mathrm{I}^{-1}\right)$. A potencialidade para formação de florações na costa

\section{AGRADECIMENTOS}

Agradecemos ao Conselho Nacional de Desenvolvimento Científico e Tecnológico (CNPq) pela concessão das bolsas de pesquisa, ao Dr. Don Anderson e Dave Kulis, de Woods Hole Oceanographic Institution pelo auxílio e disponibilização da infraestrutura necessária ao desenvolvimento do trabalho e ao pessoal do laboratório de ecologia do fitoplâncton e microorganismos marinhos da FURG pela valorosa contribuição. sul do Brasil, juntamente com os altos valores de toxicidade observados para estas cepas (Persich et al., 2006) indicam que quando ocorreu a floração na Praia do Cassino, a concentração de saxitoxinas pode ter ultrapassado os limites de segurança nos organismos filtradores locais.

Florações tóxicas de $A$. tamarense ocorrem na costa da Argentina e Uruguai há mais de 20 anos, as quais provavelmente serviram de inóculo para as cepas sul brasileiras, conforme resultados da análise genética (Persich et al., 2006). A formação de cistos de resistência, verificados no sedimento, nas proximidades da Praia do Cassino (Persich e Garcia, 2003), atua no sentido da propagação das células no ambiente, as quais podem retornar à forma vegetativa ao encontrarem novamente as condições ambientais favoráveis ao seu crescimento. Ao menos duas florações de $A$. tamarene ocorreram na Praia do Cassino, a primeira em agosto de 1996 (Odebrecht et al., 1997), e a segunda em agosto de 1997, observada no momento da obtenção da cepa C6, utilizada neste trabalho. A taxa máxima de crescimento celular obtida neste trabalho $\left(0,7 \mathrm{divdia}^{-1}\right)$ é maior do que o máximo verificado em outros locais, inclusive em áreas sujeitas a florações recorrentes da espécie. Os valores de toxicidade encontrados (66.000 fgSTX cel ${ }^{-1}$ ), também estão entre os maiores valores encontrados em cultivos da espécie. Estes altos valores de taxas de crescimento e toxicidade verificados no presente trabalho, associados à riqueza da fauna bentônica nesta área, além da verificação prévia de florações de Alexandrium tamarense na Praia do Cassino, evidenciam o potencial de ocorrência de florações tóxicas da espécie.

\section{LITERATURA CITADA}

ALLEN, JI, D ANDERSON, M BURFORD, S DYHRMAN, K FLYNN, P M GLIBERT, G EDNA, C HEIL, K SELLNER, T SMAYDA \& M ZHOU. 2006. GEOHAB. Global Ecology and Oceanography of Harmful Algal Blooms, Harmful Algal Blooms in Eutrophic Systems. In: GLIBERT (ed.) IOC and SCOR, Paris and Baltimore, 74p.

ANDERSON, DM. 1980. Effects of temperature conditioning on development and germination of Gonyaulux tamarensis (Dinophyceae) hypnozigotes. J. Phycol., 16: 166-172.

.ANDERSON, DM. 1998. Physiology and Bloom Dynamics of Toxic Alexandrium Species, with Emphasis on Life Cycle 
Transitions. In: ANDERSON, CEMBELLA \& HALLEGRAEFF, (Eds.), Physiological Ecology of Harmful Algal Blooms. Springer. 29-48..

ANDERSON, DM. 2004. The growing problem of harmful algae. Tiny plants pose a potent threat to those who live in and eat from the sea. In: http://oceanusmaga.whoi.edu/v43n1/Anderson.html.

ANDERSON, DM \& D WALL. 1978. Potential importance of benthic cysts of Gonyaulax tamarensis and $G$. excavata in initiating toxic dinoflagellate blooms. J. Phycol., 14(2): 224-234.

ANDERSON, DM., SW CHISHOLM \& CJ WATRAS. 1983. Importance of life cycle events in the population dynamics of Gonyaulax tamarensis. Mar. Biol. 76: 179-189.

ANDERSON, DM, DM KULIS \& BJ BINDER. 1984. Sexuality and cyst formation in the dinoflagellate Gonyaulax tamarensis: cyst yield in batch cultures. J. Phycol. 20: 418-425.

ANDERSON, DM, DM KULIS, GJ DOUCETTE, JC GALLAGHER \& H BALECH. 1994. Biogeography of toxic dinoflagellate in the genus Alexandrium from the Northeastern United States and Canada. Mar. Biol. 120:467-478.

ANDRINOLO, D, N SANTINELLI, S OTAÑO, V SASTRE \& N LAGOS. 1999. Paralytic shellfish toxins in mussels and Alexandrium tamarense at Valdes Peninsula, Chubut, Patagonia, Argentina: kinetics of a natural depuration. J. Shellfish Res. 18 (1): 203-209.

BALECH, E. 1995. The Genus Alexandrium Halim (Dinoflagellata). Sherkin Island Marine Station, Cork, Ireland, 151p.

BENAVIDES, H, RM NEGRI \& JI CARRETO. 1983. Investigaciones sobre el ciclo de vida del dinoflagelado toxico Gonyaulax excavata (Braarud) Balech (Dinophyceae). Physis (Buenos Aires), Secc. A, 41(101):135-142.

BRAND, L. 1980. Genetic variability and differentiation in niche components of marine phytoplankton species. PHD thesis, Woods Hole Oceanographic Institution- Massachusetts Institute of Technology Joint Program in Biological Oceanography, 227p.

BRAND, LE, RRL GUILLARD \& LS MURPHY. 1981. A method for the rapid and precise determination of acclimated phytoplankton reproduction rates. J. Plankton Res., 3:193-201.

BRAZEIRO, A, S MENDÉZ \& G FERRARI. 1997. The first toxic Bloom of Alexandrium tamarense in Uruguay: associated environmental factors. Atlântica, Rio Grande, 19: 19-29.

CALLIARI, LJ, AHF KLEIN \& FCR BARROS. 1996. Beach differentiation along the Rio Grande do Sul coastline (Southern Brazil). Revista Chilena de Historia Natural, 69: 485-493.

CARRETO, JI, RM NEGRI, HR BENAVIDES \& R AKSELMAN. 1985. Toxic dinoflagellate blooms in the Argentine Sea. pp. 147152. In: ANDERSON, WHITE \& BADEN (Eds.) Toxic Dinoflagellates. Elsevier Science Publishing Co, Inc.

CARRETO, JI, HR BENAVIDES, RM NEGRI \& PD GLORIOSO. 1986. Toxic red-tide in the Argentine Sea. Phytoplankton distribution and survival of the toxic dinoflagellate Gonyaulax excavata in a frontal area. J. Plankton Res., 8: 15-28.

CARRETO, JI, NG MONTOYA, ADC COLLEONI \& $R$ AKSELMAN. 1998. Alexandrium tamarense blooms and shellfish toxicity in the Argentine sea: a retrospective view. In: REGUERA, BLANCO, FERNÁNDEZ \& WYATT (Eds.). Harmful Algae. Xunta de Galicia and Intergovernmental Oceanographic Comission of UNESCO. 131-134.

CASTELLO, JP, AK DUARTE, O Jr MÖLLER, LF NIENCHESKI, C ODEBRECHT, G WEISS, RP HABIAGA, VR
BELLOTTO, D KITZMANN, C SOUTO, RB SOUZA, AM CIOTTI, G FILLMANN, PR SCHWINGEL, JC BERSANO, M CIRANO, $K$ FREIRE, I LIMA, R MELLO, A MONTEIRO, C Jr RESGALA, I SOARES, M SUZUKI. 1990. On the importance of coastal and subantartic waters for the shelf ecosystem off Rio Grande do Sul. Publ. Acad. Ciências Est. (S. Paulo)71: 112-129.

CHEBIB, HA, AD CEMBELLA \& D ANDERSON. 1993. Differential paralytic shellfish toxin accumulation and detoxification kinetics in transplanted populations of Mytilus edulis exposed to natural blooms of Alexadrium excavatum. 1993. In: Smayda \& Shimizu (Eds.). Toxic Phytoplankton Blooms in the Sea. Fifth International Conference on Toxic Marine Phytoplankton, Elsevier, 383-388 $\mathrm{p}$.

DALE, B. 1983. Dinoflagellate resting cysts. In: FRYXEL(Ed.). Survival strategies of the algae. Cambridge, 69-136p.

DAVISON, P \& CM YENTSCH.1985. Occurrence of toxic dinoflagellates and shellfish toxin along coastal Uruguay, South America. In: ANDERSON, WHITE \& BADEN (Eds.). Toxic Dinoflagellates, Elsevier Science Publishing Co. 153-157p.

FACHIN, S. 1998. Caracterização do perfil de equilíbrio da antepraia na costa do Rio Grande do Sul. Tese de Mestrado, Universidade Federal do Rio Grande do Sul- Curso de PósGraduação em Geociências. 114p.

FAUCHOT, J, M LEVASSEUR, $S$ ROY, R GAGNON \& A WEISE, 2005. Environmental factors controlling Alezxandrium tamarense (Dinophyceae) growth rate during a red tide event in St. Lawrence estuary (Canada). J. Phycol. 41: 263-272.

GARCIA, VMT, C ODEBRECHT \& LR RÖRIG. 1994. Florações nocivas de fitoplâncton na costa brasileira. IOC Workshop Report. 101: 9-14.

GIANUCA, NM. 1998. Benthic Beach Invertebrates. SEELIGER, ODEBRECHT \& CASTELLO (Eds.). Subtropical Convergence Environments. Springer, 114-117p.

GLIBERT, PM, DM ANDERSON, P GENTIEN, E GRANÉLI \& KG SELLNER. 2005. The global, complex phenomena of Harmful Algal Blooms. Oceanography, 18 (2): 136-147.

GUILLARD, R. R. L. \& RYTHER, J. H. 1962. Studies of marine planktonic diatoms. I Cyclotella nana Husted and Detonula confervacea (Cleve) Gran. Can. J. Microbiol., 8:229-239.

GUZMÁN, L., H PACHECO, G PIZARRO \& C ALARCÓN. 2002. Alexandrium catenella y veneno paralizante de los mariscos en Chile. In: SAR, FERRARIO \& REGUERA (Eds). Floraciones algales nocivas en el cono sur americano. 235-256p.

HAIMOVICI, M, SD PEREIRA, \& PC VIEIRA. 1989. La pesca demersal en el sur de Brasil en el periodo 1975-1985. Frente Mar (Montevideo) 5: 151-163.

HALL, S., G STRICHARTZ, E MOCZYDLOWSKI, A RAVIDRAN \& PB REICHARDT. 1990. The Saxitoxins Sources, Chemistry, and Pharmacology. In: HALL \& STRICHARTZ (Eds.). Marine Toxins Origin, Structure and Molecular Pharmacology., ACS Symposium Series 418, 29-65p.

HAMASAKI, K, M HORIE \& S TAGUCHI. 1997. Growth and toxicity of Alexandrium tamarense isolated from Hiroshima bay during blooms in 1992-1995. REGUERA, BLANCO, FERNÁNDEZ \& WYATT (Eds). Harmful Algae. VIII International Conference. 341342p.

KIM, HG, SG LEE, WA LIM, JS LEE \& JH KIM. 1996. Environmental physiology of Alexandrium tamarense isolated from Chinhae Bay, the South Sea of Korea. In: YASUMOTO, OSHIMA \& 
FUKUYO (Eds.). Harmful and Toxic Algal Blooms. UNESCO, 5760p.

MACHADO, PA. 1979. Dinoflagellate bloom on the Brazilian South Atlantic coast. In: TAYLOR \& SEELIGER (Eds.). Toxic Dinoflagellate Blooms. Elsevier, New York, 29-32p.

MATSUOKA, K \& Y FUKUYO. 1987. Manual for Modern Dinoflagellate Cyst Study. Second edition. Workshop of International Symposium on Red Tides, Takamatsu, Japan.

MÉNDEZ, S. 1995. Bivalve mortality on Southwest Atlantic shores. Harmful Algae News. No 10/11, p.12.

MÉNDEZ, S, D SEVEROV, G FERRARI \& C MESONES. 1996. Early spring Alexandrium tamarense toxic blooms in the Uruguayan waters. In: Harmful and Toxic Algal Blooms. Proc. of the $7^{\circ} \mathrm{Int}$. Conf. on Tox. Phyt., Japan. Unesco, p113-116.

MÉNDEZ, S \& G FERRARI. 2002. Floraciones algales nocivas en Uruguay: antecedentes, proyetos en curso y revisión de resultados. In: SAR, FERRARIO \& REGUERA (Eds). Floraciones algales nocivas en el cono sur americano.269-288.

MOORE, SK, VL TRAINER, NJ MANTUA, MS PARKER, EA LAWS, LC BACKER. \&LE FLEMING. 2008. Impacts of climate variability and future climate change on harmful algal blooms and human health. Environmental Health 2008, 7(Suppl 2) 12p.

ODEBRECHT, C, S MENDÉZ \& VMT GARCIA. 1997. Oceanographic processes and $\mathrm{HAB}$ in the Subtropical Southwestern Atlantic (28-36 $\left.{ }^{\circ} \mathrm{S}\right)$. VIII International Conference on Harmful algae. Vigo (Espanha) 1997. Resumo

ODEBRECHT, C, L RÖRIG, VMT GARCIA \& PC ABREU. 1995. Shellfish mortality and a red tide event in southern Brazil. In: Lassus, Arzul, Erard, Gentien \&Marcaillou (Eds.). Harmful Marine Algal Blooms. Technique et Documentation - Lavousier, Intercept Ltd. .213-218p.

PERSICH, GR \&, VMT GARCIA. 2003. Ocorrência de cistos de dinoflagelados, com ênfase em espécies potencialmente nocivas, no sedimento próximo à desembocadura da Laguna dos Patos (RS). Atlântica, Rio Grande, 25(2): 123:133.

PERSICH, GR, DM KULIS, EL LILLY, DM ANDERSON \& VMT GARCIA. 2006. Probable origin and toxin profile of Alexandrium tamarense (Lebour) Balech from southern Brazil. Harmful Algae 5: 36-44.

PRAKASH, A. 1967. Growth and toxocity of a marine dinoflagellate, Gonyaulax tamarensis. J. Fish. Res. Bd. Canada, 24(7): 1589-1606.

PREMAZZI, G \& L VOLTERRA. 1993. Microphyte Toxins, A manual for toxin detection, environmental monitoring and therapies to counteract intoxications. Commission of the European Communities, Luxembourg. 336p.

ROSA, ZJ \& TC BUSELATO. 1981. Sobre a ocorrência de floração de Gyrodinium aureolum Hulburt (Dinophyceae) no litoral do Estado do Rio Grande do Sul, Brasil. Iheringia, 28: 169-179.

SANTINELLI, N, V SASTRE \& JL ESTEVES. 2002. Episódios de algas nocivas en la Patagonia Argentina.In: SAR, FERRARIO \& REGUERA (Eds). Floraciones algales nocivas en el cono sur americano. 197-208p.

SCHOLIN, CA, M HERZOG, M SOGIN \& DM ANDERSON. 1994. Identification of group- and strain-specific genetic markers for globally distributed Alexandrium (Dinophyceae). II. Sequence analysis of a fragment of the LSU rDNA gene. J. Phycol. 30: 9991011.

SCHOLIN, CA, G HALLEGRAEFF \& DM ANDERSON. 1995. Molecular evolution of the Alexandrium tamarense "species complex" (Dinophyceae): disperal in the North American and West Pacific regions. Phycol. 34:472-485.

SENGERS, PA. 1908. Primera observación de una causa nueva de enfermidad del hígado causando una hipertrofia y cirrosis atrófica consecutivas por excesividad funcional debido a absorción de toxinas, y primera observación de esplenomgalia concomitante com hipertrofia de bazo en estas afecciones. La Semana Médica (Buenos Aires) 20: 117-119.

SHIMADA, H, T HAYASHI \& T MIZUSHIMA. 1996. Spatial distribution of Alexandrium tamarense in Funka Bay, Southwestern Hokkaiko, Japan. In: YASUMOTO, OSHIMA \& FUKUYO (Eds). Harmful and Toxic Algal Blooms. Seventh International Conference on Toxic Phytoplankton, Japan. UNESCO, 219-221p.

SU, HM, IC LIAU \& YM CHIANG. 1993. Role of temperature, salinity and ammonia on the occurrence of the Taiwanese strain of Alexandrium tamarense. In: SMAYDA \& SHIMIZU (Eds.). Toxic Phytoplankton Blooms in the Sea. Fifth International Conference on Toxic Marine Phytoplankton. Elsevier, 837-842p.

TAYLOR, FJR. 1985. The taxonomy and relationship of red tide flagellates. In: ANDERSON, WHITE \& BADEN (Eds). Toxic Dinoflagellates. 11-26p.

WATRAS, C J, SW CHISHOLM \& DM ANDERSON. 1982. Regulation of growth in an estuarine clone of Gonyaulax tamarensis: Salinity-dependent temperature responses. J. Exp. Mar. Bio. Ecol. 62: 25-37p.

WHITE, A. 1978. Salinity effects on growth and toxic content of Gonyaulax excavata, a marine dinoflagellate causing Paralytic Shellfish Poisoning. J. Phycol. 14: 475-479p.

YENTSCH, C, EJ COLE \& MG SALVAGGIO. 1975. Some of the growth chatacteristics of Gonyaulax tamarensis isolated from the Gulf of Maine. In: LOCICCERO (Ed). Toxic Dinoflagellate Blooms. Proceedings of the International Conference (1st). Massachusetts Science and Technology Foundation, Wakefield, 163-80p.

Recebido: 06/03/2009

Aceito: 12/08/2009 
